\title{
Experimental observation of whispering gallery modes in novel silicon microcylindrical resonators
}

\author{
N. Vukovic ${ }^{1}$, N. Healy ${ }^{1}$, P. Horak ${ }^{1}$, G. S. Murugan ${ }^{1}$, J. R. Sparks' ${ }^{2}$, P. J. A. Sazio ${ }^{1}$, J. V. Badding ${ }^{2}$, \\ and A. C. Peacock ${ }^{1}$ \\ ${ }^{1}$ Optoelectronics Research Centre, University of Southampton, Southampton SO17 1BJ, UK \\ ${ }^{2}$ Department of Chemistry and Materials Research Institute, Pennsylvania State University, 16802 PA, USA
}

Microresonators that support whispering gallery modes (WGMs) are ideal systems for studying nonlinear phenomena at low thresholds due to the small mode volumes and the high quality (Q) factors and, as such, they are currently generating much scientific interest [1]. A variety of geometries have been investigated including microspheres, microdisks, toroids and micropillars, using a range of dielectrics and, more recently, semiconductor materials. One of the major challenges in fabricating semiconductor microresonators is obtaining the smooth, defect-free, surfaces required for high Q operation. In this paper, we present a novel approach to fabricating high quality silicon microcylindrical resonators starting from the silicon optical fibre platform [2]. The silicon fibres are fabricated using a high pressure chemical deposition technique to fill silica capillaries with the semiconductor material. This process can be easily modified to fill capillaries of various internal diameters with the deposited material taking on the pristine smoothness of the capillary walls ( $0.1 \mathrm{~nm}$ RMS). As an optical material, silicon is particularly attractive due to its broad transparency window that extends from the telecoms band to the mid-IR $(\sim 1.2-7 \mu \mathrm{m})$, as well as its high optical damage threshold and large nonlinearities.

To fabricate our silicon microcylindrical resonators we simply etch out the semiconductor core from the silica cladding surrounding the core using a buffered hydrofluoric acid solution in a single process step. The resonator used in our investigations has an amorphous silicon (a-Si) core, with a $\sim 5.6 \mu \mathrm{m}$ diameter, which was measured (using a ZeScope 3D optical profiler) to have an exceptionally low surface roughness $\sim 0.14 \mathrm{~nm}$ RMS (compared to other silicon resonators, typically a few $\mathrm{nm}$ ), with a roughness correlation length of $\sim 123 \mathrm{~nm}$. To characterize the resonator we use a standard evanescent coupling approach (SMF fibre tapered to a diameter of 1 $-2 \mu \mathrm{m}$ ) to launch a tuneable CW source over the wavelength range $1.48-1.64 \mu \mathrm{m}$, as shown in the inset of Fig.1. The resonant features can be seen in the transmission spectrum of Fig. 1, clearly showing the excitation of different families of higher order radial and azimuthal modes. The modes are labelled with the corresponding radial $(n)$ and azimuthal $(l)$ mode numbers for the WGM resonances, as determined numerically using the coupled mode theory (CMT), and the measured Q factor. We attribute the asymmetry of the resonances to the optical bistability of the resonator and this is supported by Fig. 2, which shows that, at powers above $100 \mu \mathrm{W}$, the sweeping action of the input laser has the effect of red-shifting the resonance. The sharp increase in transmission occurs when the optically induced red-shift reaches its maximum attainable value at resonance. Although the measured Q values $\left(\sim 10^{3}\right)$ are of a similar magnitude to what has been reported in silicon microdisk resonators, they are still orders of magnitude lower than the Q's predicted via the CMT for a resonator with this value of surface roughness. Calculating all of the estimated contributions to the measured Q, we have found that the major contributions to the overall quality factor are the large mode matching between the resonator and the coupling silica taper, as well as the intrinsic material losses for the a-Si $(20 \mathrm{~dB} / \mathrm{cm}$ at $1.55 \mu \mathrm{m})$. The fabrication and coupling procedures required to obtain ultra-high Q resonators by this simple two step (deposit then etch) approach will be discussed.
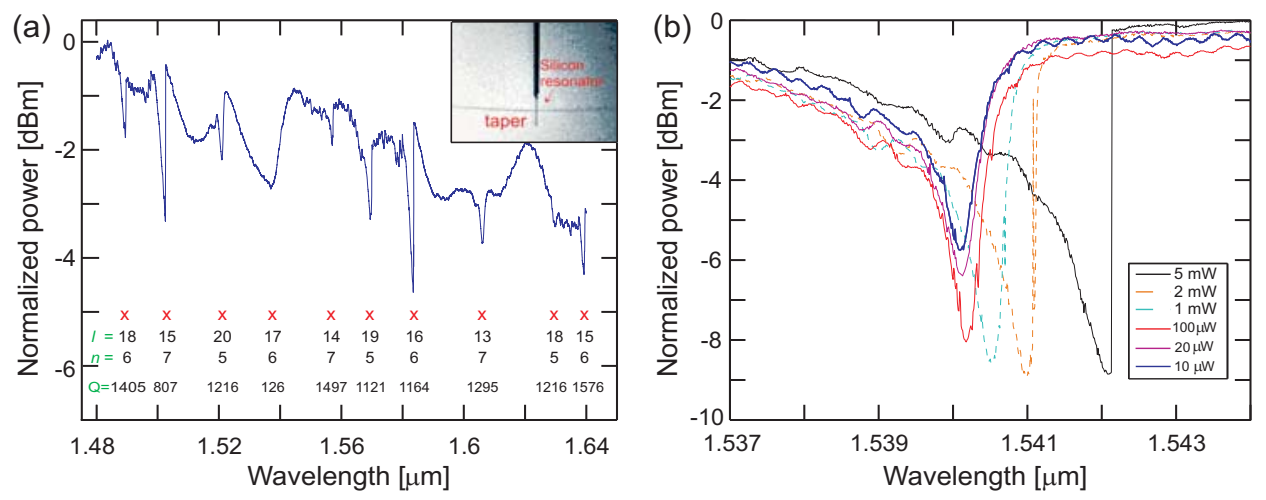

Fig. 1 (a) Silicon resonator transmission spectrum, input power $\sim 6 \mathrm{~mW}$. Inset: silicon resonator with tapered coupling fibre. (b) Transmission spectra near the $1.54 \mu \mathrm{m}$ resonance as a function of input power.

\section{References}

[1] K. Vahala, “Optical Microcavities,” Nature 424, 839 (2003).

[2] J. V. Badding, V. Gopalan, and P. J. A. Sazio, “Building Semiconductor Structures in Optical Fibers,” Phot. Spectra 40, 80 (2006). 